ISSN 0258-7122 (Print), 2408-8293 (Online)

Bangladesh J. Agril. Res. 43(1): 89-97, March 2018

\title{
RESPONSE OF STRAWBERRY TO NPKS ON YIELD IN TERRACE SOIL
}

\author{
R. AHMED ${ }^{1}$, M. R. KARIM², M. A. QUDDUS ${ }^{3}$, S. AHMED ${ }^{4}$ \\ AND M. A. SIDDIKY ${ }^{5}$
}

\begin{abstract}
Strawberry (Fragaria $X$ annanassa Duch.) is highly exhaustive and responsive to chemical fertilizers. An experiment on strawberry was conducted in fruits research field of Horticulture Research Centre, BARI, Gazipur over three consecutive years, 2009-2010, 2010-2011 and 2011-2012 to find out the suitable combination of nitrogen $(\mathrm{N})$, phosphorus $(\mathrm{P})$, potassium $(\mathrm{K})$ and sulphur $(\mathrm{S})$ for yield maximization of strawberry. Fourteen treatment combinations were tested in this trial comprising four levels each of $\mathrm{N}\left(0,90,115\right.$ and $\left.140 \mathrm{~kg} \mathrm{ha}^{-1}\right), \mathrm{P}(0,20,40$ and $\left.60 \mathrm{~kg} \mathrm{ha}^{-1}\right), \mathrm{K}\left(0,85,110\right.$ and $\left.135 \mathrm{~kg} \mathrm{ha}^{-1}\right)$ and $\mathrm{S}\left(0,15,25\right.$ and $\left.35 \mathrm{~kg} \mathrm{ha}^{-1}\right)$ with blanket dose of $4 \mathrm{~kg} \mathrm{Zn} \mathrm{ha-1,} 2 \mathrm{~kg} \mathrm{~B} \mathrm{ha}^{-1}$ and $10 \mathrm{t}$ cowdung ha- ${ }^{-1}$. The experiment was set up in randomized completely block design with three replications. Results showed that treatment combination $\mathrm{N}_{115} \mathrm{P}_{40} \mathrm{~K}_{110} \mathrm{~S}_{25}$ (underscript represent kg ha ${ }^{-1}$ ) produced higher strawberry fruit yield $\left(9.59 \mathrm{t} \mathrm{ha}^{-1}\right.$ ) followed by $\mathrm{N}_{90} \mathrm{P}_{40} \mathrm{~K}_{110} \mathrm{~S}_{25} \mathrm{~kg}$ $\mathrm{ha}^{-1}$ and $\mathrm{N}_{140} \mathrm{P}_{40} \mathrm{~K}_{110} \mathrm{~S}_{25} \mathrm{~kg} \mathrm{ha}^{-1}$ treatment combinations. The lowest fruit yield (6.05 $\left.\mathrm{t} \mathrm{ha}^{-1}\right)$ was found in control $\left(\mathrm{N}_{0} \mathrm{P}_{0} \mathrm{~K}_{0} \mathrm{~S}_{0}\right)$ treatment. Therefore, the combination of $\mathrm{N}_{115} \mathrm{P}_{40} \mathrm{~K}_{110} \mathrm{~S}_{25} \mathrm{~kg}^{-1}$ may be considered as suitable dose for strawberry cultivation in terrace soils of Bangladesh.
\end{abstract}

Keywords: Fertilizer (N, P, K and S), strawberry yield, terrace soil.

\section{Introduction}

Strawberry (Fragaria X annanassa Duch.) belongs to rose family. It is a good source of vitamin $\mathrm{C}$, folate and potassium, and is relatively low in calories. Excellent ice cream and jam or jellies are made with strawberry due to its attractiveness, tasty, pleasant aroma and flavor (Rayees et al., 2015). Strawberry has attained a premier position in the fresh fruit market and processing industries of the world (Sharma and Sharma, 2003). Although it is widely grown in temperate zones, its cultivation is also possible in the sub-tropical zones as day neutral cultivars (Asrey and Singh, 2004). Strawberry offers quicker returns than any other fruit crop.

Most of the soils and climatic conditions of Bangladesh are suitable for strawberry production. Cultivation of this fruit has started in Bangladesh. However, the average yield is lower compared to the other sub-tropical countries,

\footnotetext{
${ }^{1,2}$ Scientific Officer, Horticulture Research Centre, Bangladesh Agricultural Research Institute (BARI), Gazipur, ${ }^{3,5}$ Senior Scientific Officer, Horticulture Research Centre, BARI, Gazipur, ${ }^{4}$ Scientific Officer, Biotechnology Division, BARI, Gazipur, Bangladesh.
} 
imbalance fertilization and poor agronomic practices being one of the best reasons. On the other hand, excess use of chemical fertilizers, pesticides and herbicides renders adverse effects on soil health and environment quality (Macit et al., 2007). For sustainable crop yields, balanced fertilizations with all the nutrients (major and trace) that are deficient in soils need to be taken into account. Role of nitrogen involves in vigorous vegetative growth with dark green color. It is a constituent of protein and nucleic acids and an integral part of chlorophyll. Plant cells need to have adequate phosphorus before they divide. Phosphorus increases root growth, yield, enhances plant maturity and promotes resistance to root rot disease and winter kill (Norfleet, 1998). Potassium is associated with activation of enzymes related to starch synthesis, regulation of stomata openings, imparts disease, cold and drought resistance to plants. Sulphur is a constituent of some amino acids, biotin and coenzyme. It is involved in chlorophyll synthesis (FRG, 2012).

Farmers of Bangladesh usually use fertilizers based on their own estimate without thinking of balance fertilization which causes deterioration of soil fertility. As intensive crop cultivation is becoming more and more necessary to meet the demand of the over population, the soil nutrient balance is becoming increasingly negative and thus requiring appropriate supplement through balanced nutrient management. We do not have yet any recommended dose of fertilizers for boosting fruit yield of strawberry in the BARC published Fertilizer Recommendation Guide. Hence, the experiment was undertaken to determine the dose of $\mathrm{N}, \mathrm{P}, \mathrm{K}$ and $\mathrm{S}$ for yield maximization of strawberry in terrace soils of Bangladesh.

\section{Materials and Methods}

The experiment was done at the fruits research field of Horticulture Research Centre, BARI, Gazipur during rabi season of 2009-10, 2010-11 and 2011-12 to find out the suitable combination of $\mathrm{N}, \mathrm{P}, \mathrm{K}$ and $\mathrm{S}$ for yield maximization of

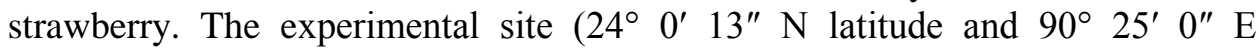
longitude) lies at an elevation of $8.4 \mathrm{~m}$ above the sea level. The Gazipur soil belongs to Chhiata series (Soil taxonomy: Udic Rhodustalf) under the agroecological zone Madhupur Tract, the soil texture being clay loam. There were 14 treatment combinations comprising four levels each of $\mathrm{N}(0,90,115$ and $140 \mathrm{~kg}$ $\left.\mathrm{ha}^{-1}\right), \mathrm{P}\left(0,20,40\right.$ and $\left.60 \mathrm{~kg} \mathrm{ha}^{-1}\right), \mathrm{K}\left(0,85,110\right.$ and $\left.135 \mathrm{~kg} \mathrm{ha}^{-1}\right)$ and $\mathrm{S}(0,15,25$ and $35 \mathrm{~kg} \mathrm{ha}^{-1}$ ). A blanket dose of fertilizer viz. $\mathrm{Zn}_{4.0} \mathrm{~B}_{2.0} \mathrm{~kg} \mathrm{ha}^{-1}$ and $10 \mathrm{t}$ ha ${ }^{1}$ cowdung were used in the trial. The arrangement of treatment combinations is shown in Table 1 . Soil samples $(0-15 \mathrm{~cm})$ before initiation of the experiment was analyzed for soil $\mathrm{pH}$ (Jackson, 1973), organic matter (Nelson and Sommers,1982), total N (Bremner and Mulvaney, 1982), exchangeable K (Jackson, 1973), exchangeable Ca \& Mg (Gupta, 2004), available P (Olsen \& Sommers, 1982), available S (Fox et al., 1964), available Zn (Lindsay and 
Norvell, 1978), available B (Page et al., 1982). The results of the soil properties are shown in Table 2.

The land was prepared thoroughly by a tractor driven siezel and rotavator. The experiment was laid out in randomized completely block design with three replications. The unit plot size was $2.5 \mathrm{~m} \times 1 \mathrm{~m}$ along with crop spacing of $50 \mathrm{~cm}$ x $50 \mathrm{~cm}$. Nitrogen, P, K, S, Zn and B were supplied as urea, TSP, MoP, gypsum, zinc sulphate and boric acid fertilizer, respectively. Zinc sulphate, boric acid and cowdung were applied to all plots at the time of final land preparation. Triple super phosphate, gypsum and 50\% MoP were added in the respective plots during final bed preparation. Fourty five days old strawberry (var. BARI Strawberry-1) seedlings were transplanted on 20 December 2009, 19 December 2010 and 21 December 2011. Urea and 50\% MoP were supplied in three equal splits after 15, 30 and 45 days of transplanting, respectively. Intercultural operations were done as and when required. Data on the number of fruits per plant, length, diameter, fruit weight per plant and individual fruit weight were recorded from eight randomly selected plants. All data on different parameters were computed for statistical analysis and the mean comparisons were adjusted by DMRT at $5 \%$ level of significance.

Table 1. Fertilizer rate wise treatment arrangement

\begin{tabular}{ccccc}
\hline Treatment & Subscripts represent kg ha $^{-1}$ & Treatment & Subscripts represent kg ha \\
\hline $\mathrm{T}_{1}$ & $\mathrm{~N}_{0} \mathrm{P}_{40} \mathrm{~K}_{110} \mathrm{~S}_{25}$ & $\mathrm{~T}_{8}$ & $\mathrm{~N}_{115} \mathrm{P}_{40} \mathrm{~K}_{0} \mathrm{~S}_{25}$ \\
$\mathrm{~T}_{2}$ & $\mathrm{~N}_{90} \mathrm{P}_{40} \mathrm{~K}_{110} \mathrm{~S}_{25}$ & $\mathrm{~T}_{9}$ & $\mathrm{~N}_{115} \mathrm{P}_{40} \mathrm{~K}_{85} \mathrm{~S}_{25}$ \\
$\mathrm{~T}_{3}$ & $\mathrm{~N}_{115} \mathrm{P}_{40} \mathrm{~K}_{110} \mathrm{~S}_{25}$ & $\mathrm{~T}_{10}$ & $\mathrm{~N}_{115} \mathrm{P}_{40} \mathrm{~K}_{135} \mathrm{~S}_{25}$ \\
$\mathrm{~T}_{4}$ & $\mathrm{~N}_{140} \mathrm{P}_{40} \mathrm{~K}_{110} \mathrm{~S}_{25}$ & $\mathrm{~T}_{11}$ & $\mathrm{~N}_{115} \mathrm{P}_{40} \mathrm{~K}_{110} \mathrm{~S}_{0}$ \\
$\mathrm{~T}_{5}$ & $\mathrm{~N}_{115} \mathrm{P}_{0} \mathrm{~K}_{110} \mathrm{~S}_{25}$ & $\mathrm{~T}_{12}$ & $\mathrm{~N}_{115} \mathrm{P}_{40} \mathrm{~K}_{110} \mathrm{~S}_{15}$ \\
$\mathrm{~T}_{6}$ & $\mathrm{~N}_{115} \mathrm{P}_{20} \mathrm{~K}_{110} \mathrm{~S}_{25}$ & $\mathrm{~T}_{13}$ & $\mathrm{~N}_{115} \mathrm{P}_{40} \mathrm{~K}_{110} \mathrm{~S}_{35}$ \\
$\mathrm{~T}_{7}$ & $\mathrm{~N}_{115} \mathrm{P}_{60} \mathrm{~K}_{110} \mathrm{~S}_{25}$ & $\mathrm{~T}_{14}$ & $\mathrm{~N}_{0} \mathrm{P}_{0} \mathrm{~K}_{0} \mathrm{~S}_{0}$ \\
\hline
\end{tabular}

Table 2. Soil properties of the experimental field

\begin{tabular}{|c|c|c|c|c|c|c|c|c|c|c|}
\hline \multirow{2}{*}{ Location } & \multirow{2}{*}{$\mathrm{pH}$} & \multirow{2}{*}{$\begin{array}{l}\mathrm{OM} \\
(\%)\end{array}$} & $\mathrm{Ca}$ & $\mathrm{Mg}$ & K & \multirow{2}{*}{$\begin{array}{c}\text { Total N } \\
(\%)\end{array}$} & $\mathrm{P}$ & $\mathrm{S}$ & $\mathrm{B}$ & $\mathrm{Zn}$ \\
\hline & & & & eq $/ 100$ & & & \multicolumn{4}{|c|}{$\mu \mathrm{g} / \mathrm{g}$} \\
\hline Joyde & 6.3 & 0.95 & 1.12 & 0. & 0.17 & 0.068 & 9 & 15 & 0.1 & 1.3 \\
\hline $\begin{array}{l}\text { Critical level } \\
\text { (FRG, 2012) }\end{array}$ & - & - & 2.0 & 0.5 & 0. & 0.1 & 10 & 10 & 0.2 & 0.6 \\
\hline
\end{tabular}

\section{Results and Discussion}

\section{Yield contributing characters}

Combination of N, P, K and S showed significant influence on yield contributing characters of strawberry in all three years (Tables $3 \& 4$ ). The number of fruits 
per plant and fruit length (mean of three years) ranged from 9.95-15.6 and 3.96$3.11 \mathrm{~cm}$, respectively. The maximum number of fruits per plant was recorded from the combination of $\mathrm{N}_{115} \mathrm{P}_{40} \mathrm{~K}_{110} \mathrm{~S}_{25}\left(\mathrm{~T}_{3}\right)$ which was significantly different from the other treatment combinations, but statistically identical with $\mathrm{T}_{2}$ and $\mathrm{T}_{4}$ treatments in every year. Result of fruit length showed a similar trend. Similar results were reported by Klaas (2000) and Funt and Blerman (2000). The minimum number of fruit per plant and the lowest fruit length were recorded from the $\mathrm{T}_{14}$ treatment $\left(\mathrm{N}_{0} \mathrm{P}_{0} \mathrm{~K}_{0} \mathrm{~S}_{0}\right)$ (Table 3$)$.

Different combination of $\mathrm{N}, \mathrm{P}, \mathrm{K}$ and $\mathrm{S}$ contributed significant role on fruit diameter and fruit weight per plant of strawberry in three following years. Mean fruit diameter varied from $2.17-3.31 \mathrm{~cm}$. The highest fruit diameter (3.31) was recorded from $\mathrm{T}_{3}$ treatment $\left(\mathrm{N}_{115} \mathrm{P}_{40} \mathrm{~K}_{110} \mathrm{~S}_{25}\right)$ followed by $\mathrm{T}_{4}$ and $\mathrm{T}_{2}$, while the minimum was recorded from the $\mathrm{T}_{14}\left(\mathrm{~N}_{0} \mathrm{P}_{0} \mathrm{~K}_{0} \mathrm{~S}_{0}\right)$. Mean fruit weight per plant varied from 145.9-237.6 $\mathrm{g}$ where the highest fruit weight per plant was found in $\mathrm{T}_{3}$ treatment $\left(\mathrm{N}_{115} \mathrm{P}_{40} \mathrm{~K}_{110} \mathrm{~S}_{25}\right)$ followed by $\mathrm{T}_{4}$ and $\mathrm{T}_{2}$. The lowest fruit diameter was observed in $\mathrm{N}_{0} \mathrm{P}_{0} \mathrm{~K}_{0} \mathrm{~S}_{0}$ treatment (Table 4). These findings are in agreement with Arancon et al. (2004), Singh and Dwivedi (2011).

Table 3. Effects of different combinations of $N, P, K$ and $S$ on fruits plant ${ }^{-1}$ and fruit length of strawberry

\begin{tabular}{|c|c|c|c|c|c|c|c|c|}
\hline \multirow{2}{*}{$\begin{array}{l}\text { Treatments } \\
\left(\mathrm{kg} \mathrm{ha}^{-1}\right)\end{array}$} & \multicolumn{4}{|c|}{ Fruits plant $^{-1}$} & \multicolumn{4}{|c|}{ Fruit length $(\mathrm{cm})$} \\
\hline & 2010 & 2011 & 2012 & mean & 2010 & 2011 & 2012 & mean \\
\hline $\mathrm{T}_{1}=\mathrm{N}_{0} \mathrm{P}_{40} \mathrm{~K}_{110} \mathrm{~S}_{25}$ & $17.5 \mathrm{def}$ & $8.33 \mathrm{gh}$ & $8.15 \mathrm{gh}$ & 11.3 & $3.26 \mathrm{e}$ & $3.09 \mathrm{~cd}$ & $3.24 \mathrm{~cd}$ & 3.20 \\
\hline $\mathrm{T}_{2}=\mathrm{N}_{90} \mathrm{P}_{40} \mathrm{~K}_{110} \mathrm{~S}_{25}$ & $19.2 \mathrm{a}-\mathrm{e}$ & $11.75 \mathrm{ab}$ & $11.55 \mathrm{ab}$ & 14.2 & $3.64 \mathrm{abc}$ & $3.82 \mathrm{abc}$ & $3.97 \mathrm{a}-\mathrm{c}$ & 3.81 \\
\hline $\mathrm{T}_{3}=\mathrm{N}_{115} \mathrm{P}_{40} \mathrm{~K}_{110} \mathrm{~S}_{25}$ & $21.8 \mathrm{a}$ & $12.55 \mathrm{a}$ & $12.35 \mathrm{a}$ & 15.6 & $3.72 \mathrm{a}$ & $4.01 \mathrm{a}$ & $4.16 \mathrm{a}$ & 3.96 \\
\hline $\mathrm{T}_{4}=\mathrm{N}_{140} \mathrm{P}_{40} \mathrm{~K}_{110} \mathrm{~S}_{25}$ & $20.4 \mathrm{abc}$ & $12.05 \mathrm{ab}$ & $11.85 \mathrm{ab}$ & 14.8 & $3.69 \mathrm{a}$ & $3.92 \mathrm{ab}$ & $4.08 \mathrm{ab}$ & 3.90 \\
\hline $\mathrm{T}_{5}=\mathrm{N}_{115} \mathrm{P}_{0} \mathrm{~K}_{110} \mathrm{~S}_{25}$ & 17.6def & $8.68 \mathrm{fgh}$ & $8.45 f-h$ & 11.6 & $3.47 \mathrm{~d}$ & $3.12 \mathrm{~cd}$ & $3.26 \mathrm{~cd}$ & 3.28 \\
\hline $\mathrm{T}_{6}=\mathrm{N}_{115} \mathrm{P}_{20} \mathrm{~K}_{110} \mathrm{~S}_{25}$ & $18.1 \mathrm{~b}-\mathrm{e}$ & $10.15 \mathrm{cde}$ & $9.95 c-e$ & 12.7 & $3.70 \mathrm{a}$ & $3.35 \mathrm{a}-\mathrm{d}$ & $3.48 \mathrm{a}-\mathrm{d}$ & 3.51 \\
\hline $\mathrm{T}_{7}=\mathrm{N}_{115} \mathrm{P}_{60} \mathrm{~K}_{110} \mathrm{~S}_{25}$ & 19.1a-e & $10.85 \mathrm{bcd}$ & $10.65 b-d$ & 13.5 & $3.56 \mathrm{bcd}$ & & $3.65 \mathrm{a}-\mathrm{d}$ & 3.58 \\
\hline $\mathrm{T}_{8}=\mathrm{N}_{115} \mathrm{P}_{40} \mathrm{~K}_{0} \mathrm{~S}_{25}$ & 19.9a-d & $9.19 \mathrm{efg}$ & $9.05 \mathrm{e}-\mathrm{g}$ & 12.7 & $3.44 \mathrm{~d}$ & $3.19 \mathrm{bcd}$ & $3.35 b-d$ & 3.33 \\
\hline $\mathrm{T}_{9}=\mathrm{N}_{115} \mathrm{P}_{40} \mathrm{~K}_{85} \mathrm{~S}_{25}$ & $20.7 \mathrm{ab}$ & $9.82 \mathrm{def}$ & $9.65 \mathrm{~d}-\mathrm{f}$ & 13.4 & $3.55 \mathrm{~cd}$ & $3.29 \mathrm{a}-\mathrm{d}$ & $3.45 \mathrm{a}-\mathrm{d}$ & 3.43 \\
\hline $\mathrm{T}_{10}=\mathrm{N}_{115} \mathrm{P}_{40} \mathrm{~K}_{135} \mathrm{~S}_{25}$ & $17.8 \mathrm{c}-\mathrm{f}$ & $11.45 \mathrm{abc}$ & $11.25 \mathrm{a}-\mathrm{c}$ & 13.5 & $3.46 \mathrm{~d}$ & $3.76 a-d$ & $3.90 \mathrm{a}-\mathrm{d}$ & 3.71 \\
\hline $\mathrm{T}_{11}=\mathrm{N}_{115} \mathrm{P}_{40} \mathrm{~K}_{110} \mathrm{~S}_{0}$ & $16.5 \mathrm{ef}$ & $9.53 \mathrm{~d}-\mathrm{g}$ & $9.35 \mathrm{~d}-\mathrm{g}$ & 11.8 & $3.65 \mathrm{abc}$ & $3.25 \mathrm{a}-\mathrm{d}$ & $3.41 \mathrm{a}-\mathrm{d}$ & 3.44 \\
\hline $\mathrm{T}_{12}=\mathrm{N}_{115} \mathrm{P}_{40} \mathrm{~K}_{110} \mathrm{~S}_{15}$ & $19.8 \mathrm{a}-\mathrm{d}$ & $11.25 \mathrm{abc}$ & $11.05 \mathrm{a}-\mathrm{c}$ & 14.0 & $3.71 \mathrm{a}$ & $3.65 \mathrm{a}-\mathrm{d}$ & $3.78 \mathrm{a}-\mathrm{d}$ & 3.71 \\
\hline $\mathrm{T}_{13}=\mathrm{N}_{115} \mathrm{P}_{40} \mathrm{~K}_{110} \mathrm{~S}_{35}$ & $19.4 \mathrm{a}-\mathrm{e}$ & $10.35 \mathrm{cde}$ & $10.35 \mathrm{c}-\mathrm{e}$ & 13.4 & $3.68 \mathrm{ab}$ & $3.43 \mathrm{a}-\mathrm{d}$ & $3.56 \mathrm{a}-\mathrm{d}$ & 3.56 \\
\hline $\mathrm{T}_{14}=\mathrm{N}_{0} \mathrm{P}_{0} \mathrm{~K}_{0} \mathrm{~S}_{0}$ & $15.3 f$ & $7.38 \mathrm{~h}$ & $7.15 \mathrm{~h}$ & 9.95 & $3.16 \mathrm{e}$ & $3.02 \mathrm{~d}$ & $3.15 \mathrm{~d}$ & 3.11 \\
\hline $\mathrm{CV}(\%)$ & 7.52 & 5.91 & 6.42 & - & 5.11 & 9.88 & 8.85 & - \\
\hline
\end{tabular}

Values within a same column with a common letter do not differ significantly $(\mathrm{p}=0.05)$ by DMRT. 
Table 4. Effects of different combinations of $N, P, K$ and $S$ on fruits diameter and fruit wt. plant ${ }^{-1}$ of strawberry

\begin{tabular}{|c|c|c|c|c|c|c|c|c|}
\hline \multirow{2}{*}{$\begin{array}{l}\text { Treatments } \\
\left(\mathrm{kg} \mathrm{ha}^{-1}\right)\end{array}$} & \multicolumn{3}{|c|}{ Fruit diameter $(\mathrm{cm})$} & & \multicolumn{3}{|c|}{ Fruit wt. plant ${ }^{-1}(\mathrm{~g})$} & \multirow[b]{2}{*}{ mean } \\
\hline & 2010 & 2011 & 2012 & $\begin{array}{c}\text { mea } \\
\mathrm{n}\end{array}$ & 2010 & 2011 & 2012 & \\
\hline $\mathrm{T}_{1}=\mathrm{N}_{0} \mathrm{P}_{40} \mathrm{~K}_{110} \mathrm{~S}_{25}$ & $2.90 \mathrm{~b}-\mathrm{e}$ & $2.32 \mathrm{gh}$ & $2.35 \mathrm{gh}$ & 2.52 & 237.3a-e & 132.6gh & $142.6 \mathrm{gh}$ & 170.8 \\
\hline $\mathrm{T}_{2}=\mathrm{N}_{90} \mathrm{P}_{40} \mathrm{~K}_{110} \mathrm{~S}_{25}$ & $2.98 b c$ & $3.21 \mathrm{ab}$ & $3.25 \mathrm{ab}$ & 3.15 & $282.7 \mathrm{abc}$ & $190.6 \mathrm{abc}$ & $199.6 \mathrm{a}-\mathrm{c}$ & 224.3 \\
\hline $\mathrm{T}_{3}=\mathrm{N}_{115} \mathrm{P}_{40} \mathrm{~K}_{110} \mathrm{~S}_{25}$ & $3.17 \mathrm{a}$ & $3.35 \mathrm{a}$ & $3.41 \mathrm{a}$ & 3.31 & $292.7 \mathrm{a}$ & 205.1a & $215.1 \mathrm{a}$ & 237.6 \\
\hline $\mathrm{T}_{4}=\mathrm{N}_{140} \mathrm{P}_{40} \mathrm{~K}_{110} \mathrm{~S}_{25}$ & $2.93 b-e$ & $3.25 \mathrm{ab}$ & $3.29 \mathrm{ab}$ & 3.16 & $284.0 \mathrm{ab}$ & $199.9 \mathrm{ab}$ & $205.9 \mathrm{ab}$ & 229.9 \\
\hline $\mathrm{T}_{5}=\mathrm{N}_{115} \mathrm{P}_{0} \mathrm{~K}_{110} \mathrm{~S}_{25}$ & $2.87 \mathrm{de}$ & $2.44 \mathrm{fgh}$ & $2.48 \mathrm{f}-\mathrm{h}$ & 2.60 & $216.7 \mathrm{de}$ & 138.4 fgh & $145.4 \mathrm{f}-\mathrm{h}$ & 166.8 \\
\hline $\mathrm{T}_{6}=\mathrm{N}_{115} \mathrm{P}_{20} \mathrm{~K}_{110} \mathrm{~S}_{25}$ & $2.96 \mathrm{bcd}$ & $2.85 b-f$ & $2.88 \mathrm{~b}-\mathrm{f}$ & 2.90 & $254.7 \mathrm{a}-\mathrm{d}$ & $158.3 \mathrm{c}-\mathrm{g}$ & $165.4 \mathrm{c}-\mathrm{g}$ & 192.8 \\
\hline $\mathrm{T}_{7}=\mathrm{N}_{115} \mathrm{P}_{60} \mathrm{~K}_{110} \mathrm{~S}_{25}$ & $2.97 \mathrm{bcd}$ & $3.02 \mathrm{a}-\mathrm{d}$ & $3.08 \mathrm{a}-\mathrm{d}$ & 3.02 & $262.7 \mathrm{a}-\mathrm{d}$ & $172.9 \mathrm{a}-\mathrm{f}$ & $180.9 \mathrm{a}-\mathrm{f}$ & 205.5 \\
\hline $\mathrm{T}_{8}=\mathrm{N}_{115} \mathrm{P}_{40} \mathrm{~K}_{0} \mathrm{~S}_{25}$ & $2.88 \mathrm{cde}$ & $2.52 \mathrm{e}-\mathrm{h}$ & $2.55 \mathrm{e}-\mathrm{h}$ & 2.65 & $231.3 \mathrm{~b}-\mathrm{e}$ & $143.4 \mathrm{fgh}$ & $152.4 \mathrm{f}-\mathrm{h}$ & 175.7 \\
\hline $\mathrm{T}_{9}=\mathrm{N}_{115} \mathrm{P}_{40} \mathrm{~K}_{85} \mathrm{~S}_{25}$ & $2.90 \mathrm{~b}-\mathrm{e}$ & $2.72 \mathrm{c}-\mathrm{g}$ & $2.75 \mathrm{c}-\mathrm{g}$ & 2.79 & 236.7 a-e & $151.3 \mathrm{~d}-\mathrm{h}$ & $160.4 d-h$ & 182.8 \\
\hline $\mathrm{T}_{10}=\mathrm{N}_{115} \mathrm{P}_{40} \mathrm{~K}_{135} \mathrm{~S}_{25}$ & $2.99 \mathrm{~b}$ & $3.15 \mathrm{abc}$ & $3.18 \mathrm{a}-\mathrm{c}$ & 3.11 & 277.3abc & $185.8 \mathrm{a}-\mathrm{d}$ & $195.8 \mathrm{a}-\mathrm{d}$ & 219.6 \\
\hline $\mathrm{T}_{11}=\mathrm{N}_{115} \mathrm{P}_{40} \mathrm{~K}_{110} \mathrm{~S}_{0}$ & $2.84 \mathrm{e}$ & $2.65 \mathrm{~d}-\mathrm{g}$ & $2.68 \mathrm{~d}-\mathrm{g}$ & 2.72 & $227.3 \mathrm{cde}$ & $147.6 \mathrm{e}-\mathrm{h}$ & $155.6 \mathrm{e}-\mathrm{h}$ & 176.8 \\
\hline $\mathrm{T}_{12}=\mathrm{N}_{115} \mathrm{P}_{40} \mathrm{~K}_{110} \mathrm{~S}_{15}$ & $2.93 b-e$ & $3.09 \mathrm{a}-\mathrm{d}$ & $3.12 \mathrm{a}-\mathrm{d}$ & 3.05 & $276.7 \mathrm{abc}$ & $178.7 \mathrm{a}-\mathrm{e}$ & $158.7 \mathrm{a}-\mathrm{e}$ & 204.7 \\
\hline $\mathrm{T}_{13}=\mathrm{N}_{115} \mathrm{P}_{40} \mathrm{~K}_{110} \mathrm{~S}_{35}$ & $2.95 \mathrm{bcd}$ & 2.92a-e & 2.95a-e & 2.94 & $260.0 \mathrm{a}-\mathrm{d}$ & $168.3 b-g$ & $175.3 \mathrm{~b}-\mathrm{g}$ & 201.2 \\
\hline $\mathrm{T}_{14}=\mathrm{N}_{0} \mathrm{P}_{0} \mathrm{~K}_{0} \mathrm{~S}_{0}$ & $2.21 \mathrm{f}$ & $2.15 \mathrm{~h}$ & $2.16 \mathrm{~h}$ & 2.17 & $194.0 \mathrm{e}$ & $118.3 \mathrm{~h}$ & $125.3 \mathrm{~h}$ & 145.9 \\
\hline $\mathrm{CV}(\%)$ & 5.90 & 6.87 & 7.25 & 6.67 & 7.65 & 9.40 & 8.45 & 8.5 \\
\hline
\end{tabular}

Values within a same column with a common letter do not differ significantly $(\mathrm{p}=0.05)$ by DMRT.

\section{Fruit yield}

Table 5 shows that combined application of NPKS fertilizers had a significant effect on individual fruit weight and fruit yield of strawberry. The highest fruit weight (mean $16.68 \mathrm{~g}$ ) and fruit yield (mean $9.59 \mathrm{t} \mathrm{ha}^{-1}$ ) were obtained with the application of $\mathrm{N}_{115} \mathrm{P}_{40} \mathrm{~K}_{110} \mathrm{~S}_{25}$ treatment $\left(\mathrm{T}_{3}\right)$ and the lowest values were noted with $\mathrm{N}_{0} \mathrm{P}_{0} \mathrm{~K}_{0} \mathrm{~S}_{0}$ treatment $\left(\mathrm{T}_{14}\right)$. The highest yield was statistically similar with the yield recorded by treatments $\mathrm{T}_{2}\left(\mathrm{~N}_{90} \mathrm{P}_{40} \mathrm{~K}_{110} \mathrm{~S}_{25}\right)$ and $\mathrm{T}_{4}\left(\mathrm{~N}_{140} \mathrm{P}_{40} \mathrm{~K}_{110} \mathrm{~S}_{25}\right)$. The maximum fruit yields produced by these treatments can be attributed to cumulative effect of the number of fruits per plant and individual fruit weight. Comparable results were reported by other workers (Koszanski et al., 2002, Mahaveer et al., 2004). 
Table 5. Effects of different combinations of N, P, K and S on fruit weight and fruit yield of strawberry

\begin{tabular}{|c|c|c|c|c|c|c|c|c|}
\hline \multirow{2}{*}{$\begin{array}{l}\text { Treatments } \\
\left(\mathrm{kg} \mathrm{ha}^{-1}\right)\end{array}$} & \multicolumn{4}{|c|}{ Individual fruit wt. (g) } & \multicolumn{4}{|c|}{ Fruit yield $\left(\mathrm{t} \mathrm{ha}^{-1}\right)$} \\
\hline & 2010 & 2011 & 2012 & mean & 2010 & 2011 & 2012 & mean \\
\hline $\mathrm{T}_{1}=\mathrm{N}_{0} \mathrm{P}_{40} \mathrm{~K}_{110} \mathrm{~S}_{25}$ & $15.85 \mathrm{ab}$ & $10.85 \mathrm{gh}$ & $12.80 \mathrm{gh}$ & 13.17 & 9.49def & $5.98 \mathrm{ef}$ & $5.95 \mathrm{ef}$ & 7.14 \\
\hline $\mathrm{T}_{2}=\mathrm{N}_{90} \mathrm{P}_{40} \mathrm{~K}_{110} \mathrm{~S}_{25}$ & $16.25 \mathrm{a}$ & $14.85 \mathrm{abc}$ & $16.80 \mathrm{a}-\mathrm{c}$ & 15.97 & $11.31 \mathrm{abc}$ & $8.15 \mathrm{ab}$ & $8.10 \mathrm{ab}$ & 9.19 \\
\hline $\mathrm{T}_{3}=\mathrm{N}_{115} \mathrm{P}_{40} \mathrm{~K}_{110} \mathrm{~S}_{25}$ & $16.29 \mathrm{a}$ & $15.90 \mathrm{a}$ & $17.85 \mathrm{a}$ & 16.68 & $11.71 \mathrm{a}$ & $8.55 \mathrm{a}$ & $8.50 \mathrm{a}$ & 9.59 \\
\hline $\mathrm{T}_{4}=\mathrm{N}_{140} \mathrm{P}_{40} \mathrm{~K}_{110} \mathrm{~S}_{25}$ & $15.81 \mathrm{ab}$ & $15.45 \mathrm{ab}$ & $17.40 \mathrm{ab}$ & 16.22 & $11.36 \mathrm{ab}$ & $8.35 \mathrm{ab}$ & $8.32 \mathrm{ab}$ & 9.34 \\
\hline $\mathrm{T}_{5}=\mathrm{N}_{115} \mathrm{P}_{0} \mathrm{~K}_{110} \mathrm{~S}_{25}$ & $14.12 \mathrm{cde}$ & $11.25 \mathrm{fgh}$ & $13.20 f-h$ & 12.86 & $8.67 f g$ & $6.18 \mathrm{def}$ & $6.15 \mathrm{~d}-\mathrm{f}$ & 7.00 \\
\hline $\mathrm{T}_{6}=\mathrm{N}_{115} \mathrm{P}_{20} \mathrm{~K}_{110} \mathrm{~S}_{25}$ & $15.52 \mathrm{ab}$ & $13.25 b-f$ & $15.20 b-f$ & 14.66 & $10.19 \mathrm{cde}$ & 7.12a-e & 7.15a-e & 8.15 \\
\hline $\mathrm{T}_{7}=\mathrm{N}_{115} \mathrm{P}_{60} \mathrm{~K}_{110} \mathrm{~S}_{25}$ & $15.70 \mathrm{ab}$ & $13.95 \mathrm{a}-\mathrm{e}$ & $15.90 \mathrm{a}-\mathrm{e}$ & 15.18 & $10.51 \mathrm{bcd}$ & $7.45 \mathrm{a}-\mathrm{e}$ & 7.48a-e & 8.48 \\
\hline $\mathrm{T}_{8}=\mathrm{N}_{115} \mathrm{P}_{40} \mathrm{~K}_{0} \mathrm{~S}_{25}$ & $13.94 \mathrm{de}$ & $11.78 \mathrm{efg}$ & $13.75 \mathrm{e}-\mathrm{g}$ & 13.16 & $7.92 \mathrm{~g}$ & $6.35 \mathrm{def}$ & $6.38 \mathrm{~d}-\mathrm{f}$ & 6.88 \\
\hline $\mathrm{T}_{9}=\mathrm{N}_{115} \mathrm{P}_{40} \mathrm{~K}_{85} \mathrm{~S}_{25}$ & $15.83 \mathrm{ab}$ & $12.88 \mathrm{c}-\mathrm{g}$ & $14.85 \mathrm{c}-\mathrm{g}$ & 14.52 & 9.46def & $6.88 \mathrm{~b}-\mathrm{e}$ & 6.90b-e & 7.75 \\
\hline $\mathrm{T}_{10}=\mathrm{N}_{115} \mathrm{P}_{40} \mathrm{~K}_{135} \mathrm{~S}_{25}$ & $14.95 \mathrm{bcd}$ & $14.45 \mathrm{a}-\mathrm{d}$ & $16.40 \mathrm{a}-\mathrm{d}$ & 15.27 & 11.09abc & $7.95 \mathrm{abc}$ & $7.95 a-c$ & 9.00 \\
\hline $\mathrm{T}_{11}=\mathrm{N}_{115} \mathrm{P}_{40} \mathrm{~K}_{110} \mathrm{~S}_{0}$ & 15.17abc & $12.45 \mathrm{~d}-\mathrm{g}$ & $14.35 \mathrm{~d}-\mathrm{g}$ & 13.99 & $9.09 \mathrm{ef}$ & $6.59 \mathrm{c}-\mathrm{f}$ & $6.62 \mathrm{c}-\mathrm{f}$ & 7.43 \\
\hline $\mathrm{T}_{12}=\mathrm{N}_{115} \mathrm{P}_{40} \mathrm{~K}_{110} \mathrm{~S}_{15}$ & $15.47 \mathrm{ab}$ & $14.15 a-d$ & $16.10 \mathrm{a}-\mathrm{d}$ & 15.24 & $11.07 \mathrm{abc}$ & $7.65 a-d$ & 7.68a-d & 8.80 \\
\hline $\mathrm{T}_{13}=\mathrm{N}_{115} \mathrm{P}_{40} \mathrm{~K}_{110} \mathrm{~S}_{35}$ & $15.96 \mathrm{ab}$ & $13.65 \mathrm{a}-\mathrm{e}$ & $15.55 \mathrm{a}-\mathrm{e}$ & 15.05 & $10.40 \mathrm{bcd}$ & $7.22 \mathrm{a}-\mathrm{e}$ & $7.25 \mathrm{a}-\mathrm{e}$ & 8.29 \\
\hline $\mathrm{T}_{14}=\mathrm{N}_{0} \mathrm{P}_{0} \mathrm{~K}_{0} \mathrm{~S}_{0}$ & $13.48 \mathrm{e}$ & $9.15 \mathrm{~h}$ & $11.10 \mathrm{~h}$ & 11.24 & $7.76 \mathrm{~g}$ & $5.18 \mathrm{f}$ & $5.20 \mathrm{f}$ & 6.05 \\
\hline $\mathrm{CV}(\%)$ & 7.63 & 7.69 & 7.9 & 7.74 & 8.99 & 9.38 & 9.95 & 9.44 \\
\hline
\end{tabular}

Values within a same column with a common letter do not differ significantly $(\mathrm{p}=0.05)$ by DMRT.

\section{Single effect of N, P, K and S on strawberry yield}

Single effects of $\mathrm{N}$ application positively increased strawberry yield during 2010, 2011 and 2012 (Table 6). The average fruit yield varied from 7.11-9.59 $\mathrm{t} \mathrm{ha}^{-1}$, where the highest yield $\left(9.59 \mathrm{t} \mathrm{ha}^{-1}\right)$ was found in $115 \mathrm{~kg} \mathrm{~N} \mathrm{ha}^{-1}$ and the lowest yield $\left(7.11 \mathrm{t} \mathrm{ha}^{-1}\right)$ in $\mathrm{N}_{0}$. The highest yield was $37 \%$ increase over $\mathrm{N}$ control. Similar results were reported by Abu-Zahra and Tahboub (2008). Results indicated that the dose above or below $115 \mathrm{~kg} \mathrm{~N}^{-1}$ rate suppressed the potential yield of strawberry. Positive effect of $\mathrm{P}$ was also noticed on yield increase of strawberry (Table 6). The average fruit yield varied from 7.00-9.59 $\mathrm{t} \mathrm{ha}^{-1}$ where the highest yield $\left(9.59 \mathrm{t} \mathrm{ha}^{-1}\right)$ was found in $40 \mathrm{~kg} \mathrm{P} \mathrm{ha}^{-1}$ and the lowest $(7.00 \mathrm{t} \mathrm{ha}$ ${ }^{1}$ ) in $\mathrm{P}_{0}$. Strawberry yield increased with the increasing level of phosphorus as reported by Yusuf et al. (2003) and Mohamed et al. (2011). Different levels of K application also demonstrated positive influence on strawberry yield (Table 6). The average fruit yield ranged from 6.88-9.59 $\mathrm{t} \mathrm{ha}^{-1}$, the highest yield (38\% yield increase over $\mathrm{K}$ control) was noted in $110 \mathrm{~kg} \mathrm{~K} \mathrm{ha}^{-1}$ and the lowest $\left(6.88 \mathrm{t} \mathrm{ha}^{-1}\right)$ yield was in $K_{0}$. The average fruit yield due to $S$ effect was from 7.43-9.59 $t$ ha ${ }^{1}$ (Table 6). The highest yield which was $29 \%$ yield increase over S control was 
observed in $25 \mathrm{~kg} \mathrm{~S} \mathrm{ha}^{-1}$ and the lowest $\left(7.43 \mathrm{t} \mathrm{ha}^{-1}\right)$ yield was recorded from $\mathrm{S}_{0}$. The findings were corroborated by Klaas (2000) and Yadav et al. (2010).

Table 6. Single effect of N, P, K and $S$ on the yield of strawberry

\begin{tabular}{|c|c|c|c|c|c|c|c|c|}
\hline \multirow{2}{*}{$\begin{array}{c}\text { Nutrient } \\
\text { levels }(\mathrm{kg} / \mathrm{ha})\end{array}$} & \multicolumn{4}{|c|}{ Fruit yield $\left(\mathrm{t} \mathrm{ha}^{-1}\right)$} & \multicolumn{4}{|c|}{$\%$ yield increase over control } \\
\hline & 2010 & 2011 & 2012 & mean & 2009-10 & 2010-11 & 2011-12 & mean \\
\hline \multicolumn{9}{|c|}{ N level } \\
\hline 0 & 9.49 & 5.90 & 5.95 & 7.11 & - & - & - & - \\
\hline 90 & 11.31 & 8.15 & 8.10 & 9.19 & 19 & 38 & 36 & 31 \\
\hline 115 & 11.71 & 8.55 & 8.50 & 9.59 & 23 & 45 & 43 & 37 \\
\hline 140 & 11.36 & 8.35 & 8.32 & 9.34 & 20 & 42 & 40 & 34 \\
\hline \multicolumn{9}{|c|}{ P level } \\
\hline 0 & 8.67 & 6.18 & 6.15 & 7.00 & - & - & - & - \\
\hline 20 & 10.19 & 7.12 & 7.15 & 8.15 & 17 & 15 & 16 & 16 \\
\hline 40 & 11.71 & 8.55 & 8.50 & 9.59 & 35 & 38 & 38 & 37 \\
\hline 60 & 10.51 & 7.45 & 7.48 & 8.48 & 21 & 21 & 22 & 21 \\
\hline \multicolumn{9}{|c|}{ K level } \\
\hline 0 & 7.92 & 6.35 & 6.38 & 6.88 & - & - & - & - \\
\hline 85 & 9.47 & 6.88 & 6.90 & 7.75 & 19 & 8 & 8 & 12 \\
\hline 110 & 11.71 & 8.55 & 8.50 & 9.59 & 47 & 35 & 33 & 38 \\
\hline 135 & 11.09 & 7.95 & 7.92 & 8.99 & 40 & 25 & 24 & 30 \\
\hline \multicolumn{9}{|c|}{ S level } \\
\hline 0 & 9.09 & 6.59 & 6.62 & 7.43 & - & - & - & - \\
\hline 15 & 11.07 & 7.65 & 7.68 & 8.80 & 21 & 16 & 16 & 18 \\
\hline 25 & 11.71 & 8.55 & 8.50 & 9.59 & 28 & 30 & 28 & 29 \\
\hline 35 & 10.40 & 7.22 & 7.25 & 8.29 & 14 & 10 & 10 & 11 \\
\hline
\end{tabular}

\section{Conclusion}

The combination of $\mathrm{N}_{15} \mathrm{P}_{40} \mathrm{~K}_{110} \mathrm{~S}_{25}$ with blanket dose of $\mathrm{Zn}_{4.0} \mathrm{~B}_{2.0} \mathrm{~kg} \mathrm{ha}^{-1}$ and $10 \mathrm{t}$ $\mathrm{ha}^{-1}$ cowdung gave higher yield of strawberry. Thus, combined application of $\mathrm{N}, \mathrm{P}, \mathrm{K}$ and $\mathrm{S}$ at $115,40,110$ and $25 \mathrm{~kg} \mathrm{ha}^{-1}$ can be recommended for yield maximization of strawberry in terrace soil of Madhupur Tract (AEZ 28).

\section{References}

Abu-Zahra, T.R. and A.A. Tahboub. 2008. Strawberry (Fragaria x Anansa Dutch) growth, flowering and yielding as affected by different organic matter sources. Intern .J. of Botany. 4 (4):481-485. 
Arancon N.Q., C.A. Edwards, P. Bierman, C. Welch, J.D. Metzger. 2004. Influences of vermicompost on field strawberries: 1. Effects on growth and yields. Bioresource Technology. 93: 145-153.

Asrey R. and Singh R. 2004. Evaluation of strawberry varieties under semi-arid irrigated region of Punjab. Indian Journal of Horticulture 61(2): 122-124.

Bremner, J.M. and C.S. Mulvaney.1982. Total nitrogen, In: Methods of Soil Analysis, Part 2, $2^{\text {nd }}$ Ed., Page, A.L., Miller, R.H., Keeney, D.R., Am. Soc. Agron. Madison, USA. Pp. 599-622.

FRG. 2012. Fertilizer Recommendation Guide. Bangladesh Agricultural Research Council, Farmgate, Dhaka.

Funt, R.C. and P. Blerman. 2000. Composted yard waste improves strawberry soil quality and soil water relation. Acta Horticulture, 517: 37-39.

Fox, R.L., R.A. Olsen and H.F. Rhoades. 1964. Evaluating the sulphur status of soil by plant and soil test. Soil Sci. Soc. Am. Proc. 28: 243-246.

Gupta, P.K. 2004: Soil, Plant, Water and Fertilizer Analysis. Department of Agricultural Chemistry and Soil Science, Maharana Pratap University of Agriculture \& Technology, Rajasthan, India. Pp. 168-170.

Jackson, M.L. 1973. Soil Chemical Analysis. Prentice Hall of India Private Limited, New Delhi. Pp. 498.

Klaas L. 2000. Effect of manure to the growth and forming runners in strawberry. Proceedings of the International Conference on fruit Production and fruit breeding. Tatru, Estonia, 12-13 September.

Koszanski, Z., S. Karczmarczyk, E. Rumasz and B. Herman. 2002. Influence of drip irrigation and mineral fertilization on strawberry yield. Acta Agril., 90: 77-80.

Lindsay, W.L. and W.A. Norvell. 1978. Development of a DTPA soil test for Zn, Fe, Mn and Cu. Soil Sci. Soc. Am. J. 42: 421-428.

Macit I., A. Koc, S. Gule and I. Deligoz. 2007. Yield, quality and nutritional status of organically and conventionally grown strawberry cultivars. Asian Journal of Plant Sciences, 6(7): 1131-1136.

Mahaveer, P., M. P. Sharma and A. Alok. 2004. Effect of arbuscular mycorrhizal fungi and phosphorus fertilization on the post vitro growth and yield of micropropagated strawberry grown in a sandy loam soil. Canadian J. Bot., 82(3): 322-328.

Mohamed, R. A., H. Abd El-Aal and M.G. Abd El-Aziz. 2011. Effects of phosphorus, zinc and their interactions on vegetative growth characters, yield and fruit quality of strawberry. J. Hort. Sci., 3(2): 106-114.

Nelson, D.W. and L.E. Sommers. 1982. Total carbon, organic carbon and organic matter. In: Methods of Soil Analysis. Part 2. $2^{\text {nd }}$ Edition. Page, A..L, Miller, R.H., Keeney, D.R. Am. Soc. of Agron. Madison, USA. Pp. 539-580.

Norfleet, M. L. 1998. Phosphorus in agriculture. J. Environ. Qual., 25:1221-1229.

Olsen, S.R. and L.E. Sommers. 1982. Phosphorus. In: Methods of Soil Analysis, Part 2. $2^{\text {nd }}$ Edition. Page, A.L., Miller, R.H., Keeney, D.R., Am. Soc. Agron. Madison, USA. Pp. 403-427. 
Page, A.L., Miller, R.H., Keeney D.R. (eds.). 1982. Methods of Soil Analysis. Part 2. Chemical and microbiological properties. $2^{\text {nd }}$ Edition. Agronomy series 9 ASA, SSSA. Madison Wis. USA.

Rayees A. Wani, S.A. Hakeem, Sabiya Bashir, Seerat Geelani, M. N. Mughal and V.M. Prasad. 2015. Impact of integrated nutrient management on growth, yield and quality of strawberry (Fragaria $x$ annanassa Duch.) cultivation in India. Nature and Science, 13(1): 39-44.

Sharma V.P. and Sharma R.R. 2003. The Strawberry. Indian Council of Agricultural Research, New Delhi, Pp. 166.

Singh N. and H. Dwivedi. 2011. Studies on the different mulches on vegetative growth of strawberry (Fragaria $\mathrm{x}$ annanassa Duch.) cv. Chandler. Progressive Horticulture, 43(1):134-136.

Yadav, S.K., U.U. Khokhar and R.P. Yadav. 2010. Integrated nutrient management for strawberry cultivation. Indian Journal of Horticulture, 67(4): 445-449.

Yusuf, A., I. Masood, S. Z. A. Shah and M.J. Ahmed. 2003. Effect of different combinations of nitrogen, phosphorous and farm yard manure on yield and quality of strawberry. Sarhad J. Agric., 19(2): 185-188. 
